\title{
Recomendación a favor de adelantar la edad de inicio del tamizaje del cáncer de colon en los EE.UU.
}

\author{
Recommendation in favour of advancing the age of onset for colon cancer screening in the US
}

\section{Comentado de:}

US Preventive Services Task Force. JAMA 2021; 325(19):19651977. PMID: $34003218^{1}$

\section{Introducción}

El cáncer de colon y recto (CCR) es una de las causas principales de morbimortalidad en los EE.UU., y en últimos años se observó un aumento en la incidencia de esta patología en las personas menores de 50 años.

\section{Objetivos}

Revisar la evidencia disponible acerca del tamizaje del CCR para actualizar la recomendación de la Fuerza de Tareas de Servicios Preventivos de los EE.UU. (USPSTF, por sus iniciales en inglés).

\section{Población}

Adultos mayores de 45 años de edad, asintomáticos, con riesgo promedio de desarrollar CCR.

\section{Métodos}

Se realizó una revisión sistemática acerca de los beneficios y los daños asociados con el tamizaje de CCR. La búsqueda se restringió a estudios publicados en idioma inglés y en países desarrollados.

Además, se llevó a cabo un análisis de decisión basado en tres modelos de microsimulación para evaluar cuáles eran las estrategias de tamizaje más eficientes en términos de años de vida ganados versus número de estudios realizados, incorporando información epidemiológica reciente.

\section{Evaluación de la evidencia}

El tamizaje reduce la mortalidad específica por CCR. Esta evidencia proviene de ensayos clínicos aleatorizados (ECA) y estudios de cohorte prospectiva con un gran número de participantes. No se realizaron meta-análisis debido a que el número de estu- dios era limitado y su heterogeneidad era elevada.

Los principales daños asociados a la videocolonoscopía (VCC) fueron la perforación y el sangrado mayor, con una incidencia de 14,6 y 3,1 eventos cada 10.000 colonoscopías de tamizaje. Para la colonoscopía virtual por tomografía los daños directos incluyen la exposición a la radiación (valores informados entre 0,8 y 5,3 $\mathrm{mSV}$ ). Los hallazgos extracolónicos fueron comunes, pero debido a la falta de información a largo plazo, es difícil clasificarlos como daños o beneficios colaterales de este método diagnóstico.

En el estudio de microsimulación, el comienzo a los 45 años de edad fue más eficiente que a los 50 . La eficiencia del tamizaje disminuía luego de los 75 años.

\section{Recomendaciones}

La USPSTF concluye, con alta certeza, que hay un beneficio sustancial del tamizaje del CCR en adultos entre 50 y 75 años de edad.

Además concluye, con moderada certeza, que entre los 45 y los 49 años, el beneficio neto de esta intervención es moderado, mientras que entre los 75 y los 85 , en personas que alguna vez realizaron tamizaje previo, el beneficio neto es pequeño.

Las modalidades recomendadas de rastreo de esta enfermedad incluyen la prueba de sangre oculta en materia fecal (SOMF) de guayaco o inmunohistoquímica anual, la colonoscopía cada 10 años y la colonoscopía virtual (por tomografía) cada 5 años, entre otras.

\section{Conclusiones}

La USPSTF mantiene su recomendación a favor de tamizaje del CCR por distintos métodos en adultos asintomáticos de 50 a 75 años (recomendación tipo A) y de 75 a 85 años no tamizados previamente (recomendación tipo $\mathrm{C}$ ), y la extiende a las personas de 45 a 50 años (recomendación tipo B), en vistas del aumento en la incidencia de esta enfermedad detectado en este grupo etario en los últimos años.

\section{Comentario}

La USPSTF reafirma sus recomendaciones de 2016 y amplía el rango etario incluyendo pacientes de 45 a 50 años ${ }^{1}$, en consonancia con otras guías estadounidenses de publicación reciente ${ }^{2,3}$. Este consejo se fundamenta en el aumento de la incidencia del CCR documentado en este grupo etario, fenómeno que también se ha observado en otros países de altos ingresos ${ }^{4}$.

En Argentina, el Instituto Nacional del Cáncer recomienda esta práctica preventiva entre los 50 y 75 años en las personas con riesgo promedio, mediante la realización de SOMF anual ${ }^{5}$. En la actualidad no sabemos si la recomendación de adelantarlo a edades más tempranas es extrapolable a nuestro país. Para ello se necesitarían estudios locales que comprueben un aumento similar de la incidencia del CCR en adultos menores de 50 años y análisis de costo-efectividad favorables para esta intervención.

Cabe destacar que la adherencia de la población a esta práctica en el rango etario recomendado en la actualidad es menor a la del tamizaje de otros tipos de cáncer ${ }^{6}$, lo cual puede atribuirse, en parte, al desconocimiento de la población acerca de la patología y a la baja disponibilidad de sus métodos de rastreo ${ }^{7}$.

A diferencia de las recomendaciones generadas por la USPSTF, una guía internacional recientemente publicada incorpora el riesgo individual de desarrollar cáncer en la toma de decisiones, además de evidencia acerca de las preferencias de los pacientes ${ }^{8,9}$. Sus autores concluyen que la mayoría de las personas elegiría no realizar tamizaje con un riesgo de cáncer menor al $3 \%$ en 15 años de seguimiento, lo cual abarca a una gran proporción de los menores de 60 años de edad. Si bien el método que los autores utilizan para evaluar los valores y preferencias tiene limitaciones, se plantea la necesidad de explorar las preferencias individuales y poblacionales en relación al tamizaje de esta patología ${ }^{9}$. 


\section{Conclusiones del comentador}

No sabemos aún si el cambio en las recomendaciones de la USPSTF acerca de la edad de inicio del rastreo del CCR es aplicable a la población argentina.

Se destaca la necesidad de seguir ofreciendo las distintas opciones de tamizaje de esta enfermedad (incluyendo la opción de no realizarlo) a las personas de riesgo promedio de desarrollarlo, entre los 50 y los 75 años de edad, proveyendo información de alta calidad y teniendo en cuenta sus valores y preferencias.

Sguiglia S. Recomendación a favor de adelantar la edad de inicio del tamizaje del cáncer de colon en los EE.UU. . Evid Actual Pract Ambul. 2021;24(2):e002134. Available from: https://dx.doi.org/10.51987/EVIDENCIA.V24I3.6954. Comentado de: US Preventive Services Task Force. Screening for Colorectal Cancer: US Preventive Services Task Force Recommendation Statement. JAMA 2021; 325(19):1965-1977. PMID: 34003218

\section{Referencias}

1. US Preventive Services Task Force. Screening for Colorectal Cancer: US Preventive Services Task Force Recommendation Statement. JAMA. 2021;315(23):2564-2575. Available from: 10.1001/jama.2021.6238;https://jamanetwork.com/journals/jama/fullarticle/2779985.

2. Rex D, Boland CR, Dominitz JA, et al. Colorectal cancer screening: Recommendations for physicians and patients from the U.S. Multi-Society Task Force on Colorectal Cancer. Am J Gastroenterol. 2017;86(1):18-33. Available from: 10.1016/j.gie.2017.04.003;https://dx.doi.org/10.1016/j.gie.2017. 04.003.

3. Wolf AMD, Fontham ETH, Church TR, et al. Colorectal cancer screening for average-risk adults: 2018 guideline update from the American Cancer Society. CA Cancer J Clin. 2018;68(4):250-281. Available from: 10.3322/caac.21457; https://acsjournals.onlinelibrary.wiley.com/doi/full/10.3322/ caac.21457.

4. Siegel RL, Torre LA, Soerjomataram I, et al. Global patterns and trends in colorectal cancer incidence in young adults. Gut. 2019;68(12):2179-2185. Available from: 10.1136/gutjnl-2019-319511;https://dx.doi.org/10.1136/gutjnl-2019-319511.

5. Gualdrini U, lummato L, Bidart M, et al. Guía para equipos de atención primaria de la salud: información para la prevención y detección temprana del cáncer colorrectal. 1st ed. and others, editor. Ciudad Autónoma de Buenos Aires: Instituto Nacional del Cáncer; 2015. Available from: https: //sage.org.ar/wp-content/uploads/2019/05/PDF-guia-INC-CCR.pdf.

6. Argentina Ministerio de Salud y Desarrollo Social Dirección Nacional de Promoción de la Salud y Control de Enfermedades Crónicas No Transmisibles. 4ta Encuesta Nacional de Factores de Riesgo; 2018. Available from: https://bancos.salud.gob.ar/sites/default/files/2020-01/4ta-encuestanacional-factores-riesgo 2019 principales-resultados.pdf.

7. Argentina Ministerio de Salud de la Nación Instituto Nacional del Cáncer. Imágenes Sociales Del Cáncer: Estudio Nacional Para Orientar La Comunicación Social. 1st ed. and others, editor. Ciudad Autónoma de Buenos Aires -: Instituto Nacional del Cáncer; 2013. Available from: https: //bancos.salud.gob.ar/recurso/imagenes-sociales-del-cancer-estudio-nacional-para-orientar-la-comunicacion-social.

8. Lise LM, Vandvik PO, Jodal HC, et al. Colorectal cancer screening with faecal immunochemical testing. BMJ. 2019;367:I5515. Available from: https://doi.org/10.1136/bmj.I5515; https://www.bmj.com/content/367/bmj.I5515.

9. Franco JVA. Nuevas recomendaciones para el rastreo de cáncer colorrectal. Comentado de: HelsingenLM, et al. Colorectal cancer screening with faecal immunochemical testing, sigmoidoscopy or colonoscopy: a clinical practice guideline. BMJ 2019; 367:I5515. PMID: 31578196. Evid Actual Pract Ambul. 2020;22(4):e002024. Available from: 10.51987/evidencia.v22i4.4252;http://www.evidencia.org.ar/index.php/Evidencia/article/ view/4252. 